\title{
A importância do cuidado farmacêutico na prevenção e tratamento de doenças cardiovasculares
}

\author{
The importance of pharmaceutical care in the prevention and treatment of cardiovascular \\ diseases
}

\begin{abstract}
La importancia de la atención farmacéutica en la prevención y tratamiento de las enfermedades cardiovasculares
\end{abstract}

Leonardo Coutinho Ribeiro ${ }^{1 *}$.

\begin{abstract}
RESUMO
Objetivo: Apresentar as demandas relativas à atividade clínica do farmacêutico na prevenção e tratamento de doenças cardiovasculares, por meio de uma revisão narrativa que destaca os benefícios da participação deste profissional nas equipes de cuidados de saúde. Revisão Bibliográfica: As doenças cardiovasculares (DCVs) são responsáveis, anualmente, por milhões de óbitos em todo o mundo. A elevação da expectativa de vida e da incidência dos fatores de risco cardiovascular, como diabetes e a hipertensão arterial não controlada, representam os principais motivos do crescente aumento das DCVs nos últimos anos. O farmacêutico, por ser capacitado para identificar e solucionar problemas relacionados ao uso de medicamentos, pode oferecer significativa contribuição à equipe multiprofissional de saúde no que tange aos cuidados e segurança do paciente. Considerações finais: Embora a atuação clínica do farmacêutico na prevenção e tratamento de DCVs ainda seja discreta nos serviços brasileiros de saúde, é possível constatar que a participação do referido profissional nas equipes multiprofissionais de cuidados de saúde pode melhorar a qualidade da assistência aos pacientes, contribuindo para a redução da incidência de erros relacionados à terapia medicamentosa e na melhoria dos resultados terapêuticos relativos à segurança e eficácia dos medicamentos.
\end{abstract}

Palavras-chave: Assistência farmacêutica, Conciliação de medicamentos, Sistema cardiovascular.

\begin{abstract}
Objective: To present the demands applied to the pharmacist's clinical activity in the prevention and treatment of cardiovascular diseases, through a narrative review that offers the benefits of the participation of this professional in health care staff. Bibliographic Review: Cardiovascular diseases (CVDs) are responsible for millions of deaths around the world. The increase in life expectancy and incidence of cardiovascular risk factors such as diabetes and uncontrolled high blood pressure, represent the main reasons for the increasing of CVDs in the last years. The pharmacist, who is able to identify and solve problems related to medication use, can offer significant contribution to the multi-professional health staff as well as patient care and safety. Final considerations: Although the performance of pharmaceutical clinic in the prevention and treatment of CVDs is still recent in Brazilian health services, it is possible to verify that the participation of this mentioned professional in health care multi-professional staffs can improve the quality of patient care, contributing to reduction in the incidence of errors related to drug therapy and in the improvement of therapeutic results related to the safety and efficiency of medicines.
\end{abstract}

Keywords: Pharmaceutical services, Medication reconciliation, Cardiovascular system.

\section{RESUMEN}

Objetivo: Presentar las demandas relacionadas con la actividad clínica del farmacéutico en la prevención y tratamiento de enfermedades cardiovasculares, a través de una revisión narrativa que destaca los beneficios de la participación de este profesional en los equipos de atención médica. Revisión Bibliográfica: Las enfermedades cardiovasculares (ECVs) son responsables, anualmente, de millones de muertes en todo el

${ }^{1}$ Hospital Universitário Cassiano Antônio Moraes (HUCAM) / Universidade Federal do Espírito Santo (UFES). Vitória - ES. *E-mail: leeovit@yahoo.com.br 
mundo. El aumento de la esperanza de vida y la incidencia de factores de riesgo cardiovascular, como la diabetes y la hipertensión arterial no controlada, son las principales razones del aumento creciente de las ECVs en los últimos años. El farmacéutico, al poder identificar y solucionar problemas relacionados con el uso de medicamentos, puede ofrecer una contribución significativa al equipo de salud multiprofesional en términos de atención y seguridad del paciente. Consideraciones finales: Aunque la actuación clínica del farmacéutico en la prevención y el tratamiento de las ECVs todavía es reciente en los servicios de salud brasileños, es posible verificar que la participación de este profesional en equipos de atención médica multiprofesionales puede mejorar la calidad de la atención al paciente, contribuyendo a la reducción de la incidencia de errores relacionados con el tratamiento medicamentoso y en la mejora de los resultados terapéuticos relacionados con la seguridad y la eficacia de los medicamentos.

Palabras clave: Servicios farmacéuticos, Conciliación de medicamentos, Sistema cardiovascular.

\section{INTRODUÇÃO}

As doenças cardiovasculares (DCVs) constituem um dos grandes desafios da saúde mundial. Estima-se que as DCVs sejam responsáveis por mais de 17 milhões de mortes/ano no mundo, o que corresponde a mais de $30 \%$ do total de óbitos do planeta (OPAS, 2017). No Brasil, os distúrbios do sistema circulatório, além de ocuparem o posto principal de causa mortis, também se destacam entre os maiores motivos de internação hospitalar, o que gera altos custos para o sistema de saúde brasileiro (BRANT LCC, et al., 2017).

A elevação da expectativa de vida e da incidência dos fatores de risco cardiovascular, como diabetes e hipertensão arterial não controlada, representam os principais motivos do crescente aumento das DCVs no Brasil nos últimos anos, pois, se por um lado, os avanços científicos no combate às doenças e o aprimoramento dos cuidados relacionados à saúde têm contribuído para ampliar a longevidade da população mundial, em contrapartida, o aumento da idade, por si só, expande o risco do desenvolvimento de doenças crônicas, especialmente as cardiovasculares, cuja incidência dobra, em média, a cada década de vida nos adultos (PEREIRA JC, et al., 2008).

A escassez de médicos cardiologistas nas unidades de atendimento básico e hospitalar associada à grande demanda de tarefas, particularmente nos serviços públicos de saúde, requerem a colaboração de profissionais de saúde não médicos como um meio eficiente e rentável para melhorar a qualidade assistencial dos pacientes (DUNN SP, et al., 2015).

O farmacêutico, por ser capacitado para identificar e solucionar problemas relacionados ao uso de medicamentos, pode oferecer significativa contribuição à equipe multiprofissional de saúde no que tange ao acompanhamento da farmacoterapia de pacientes internados e ambulatoriais, colaborando para a otimização da terapia farmacológica, identificação de efeitos adversos e interações medicamentosas, educação ao paciente, desenvolvimento de autonomia e responsabilidade no autocuidado com a saúde e identificação prévia de erros de prescrição e utilização de medicamentos (KUO GR, et al., 2013).

Este artigo visa apresentar as demandas relativas à atividade clínica do farmacêutico na prevenção e tratamento de DCVs, por meio de uma revisão narrativa que destaca os benefícios da participação ativa deste profissional nas equipes de cuidados de saúde. Os serviços farmacêuticos de conciliação medicamentosa, educação em saúde e revisão da farmacoterapia, com ênfase na adesão ao tratamento, constituíram o foco de abordagem da presente revisão, tendo em vista a grande importância desses serviços para o seguimento clínico dos pacientes.

\section{REVISÃO BIBLIOGRÁFICA}

\section{Conciliação de medicamentos na admissão e alta hospitalares}

A conciliação de medicamentos é um serviço que visa aumentar a segurança da farmacoterapia através da obtenção de uma lista completa e precisa de todos os medicamentos que o paciente utiliza, com suas concentrações e doses correspondentes, frequência de uso, vias de administração, apresentação comercial, duração do tratamento e outras informações pertinentes (BRASIL, 2016b). 
Nas transições de cuidados assistenciais de saúde, a conciliação constitui uma etapa indispensável do processo, visto que possibilita a comparação entre a lista completa de medicamentos que o paciente usava anteriormente à transição de cuidados e as medicações prescritas após a transição (SANCHÉS OD, et al., 2009). As discrepâncias identificadas nesse processo e não justificadas pela equipe médica são consideradas erros de conciliação (CEBRIÁN BR, et al., 2016). Nos eventos clínicos de comprometimento cardiovascular agudo, o paciente é quase sempre levado às pressas ao serviço de assistência e sem condições de fornecer informações, muitas vezes acompanhado de um prestador de socorro que se encontrava junto ao paciente na ocorrência do evento cardiovascular, mas não conhece a história das doenças, nem a farmacoterapia domiciliar do afetado (BORGES EP e MACEDO AF, 2011).

Nessas situações, é imprescindível identificar um familiar ou cuidador que conheça bem o paciente e tenha condição de fornecer informações relevantes ao processo de conciliação. Caso esse familiar ou cuidador não possa se apresentar pessoalmente ao serviço de assistência, a instituição deve viabilizar formas alternativas para que as informações sejam fornecidas por meio de aplicativos de dipositivo móvel ou correio eletrônico, preferencialmente, acompanhadas de imagens digitalizadas ou fotografias das prescrições e/ou medicamentos, que garantam a fidelidade das informações prestadas (RUIZ BSJ, et al., 2016).

É importante que o farmacêutico esteja envolvido em todas atividades que visem assegurar a efetividade da conciliação, colhendo informações por meio de entrevistas aos assistidos e acompanhantes e examinando receituários médicos e medicamentos dos pacientes (RUIZ BSJ, et al., 2016). Uma conciliação de medicamentos minuciosa pode evitar erros de posologia ou vias de administração, duplicidade de prescrição, omissão de farmacoterapia necessária e associações medicamentosas inadequadas.

A partir de um estudo observacional descritivo realizado numa unidade hospitalar de acidentes vasculares cerebrais, os autores constataram que, no momento da admissão dos doentes, houve omissão de medicamentos em $80,9 \%$ dos casos em que os pacientes foram tratados com cinco ou mais medicamentos, o que indica a subutilização de medicamentos em pacientes internados com doença aguda (BORGES EP e MACEDO AF, 2011).

As várias demandas relacionadas ao diagnóstico e tratamento das DCVs e prevenção de complicações, associadas ao número insuficiente de médicos cardiologistas nas unidades hospitalares, em especial, nos serviços públicos de saúde, podem provocar uma certa desatenção aos outros agravos do paciente aparentemente menos importantes, resultando na omissão do registro de informações durante a anamnese. Estima-se que, no momento da admissão, erros de conciliação ocorram em mais de $50 \%$ dos pacientes hospitalizados com quadros clínicos agudos (RUIZ BSJ, et al., 2016), sendo a omissão de drogas um dos erros mais frequentes (JUVANY R e JÓDAR R, 2012).

O serviço de farmácia clínica deve elaborar, com base na literatura científica e documentos validados, um instrumento de coleta de dados para ser aplicado nas primeiras 24 horas de ingresso do assistido no serviço e que possibilite extrair dos entrevistados, pacientes ou acompanhantes, as informações pertinentes à conciliação de medicamentos (MACHUCA M, et al., 2004).

Em pacientes pré-operatórios de cirurgias de revascularização do miocárdio, por exemplo, a identificação de todos os medicamentos de uso domiciliar, inclusive os decorrentes de automedicação, exibe grande relevância, visto que algumas classes de fármacos, como anticoagulantes, antiagregantes plaquetários e hipoglicemiantes orais devem ter seu uso suspenso por dias ou horas de antecedência ao procedimento, a fim de evitar complicações hemorrágicas ou episódios de acidose lática (GARBERO RF e VIEIRA LA, 2007).

Em razão da visão equivocada e popularmente disseminada de que os produtos naturais não apresentam contraindicações, alguns indivíduos podem considerar desnecessário informar, na admissão hospitalar, que usam fitoterápicos. Entretanto, alguns desses medicamentos, como Ginseng (Panax ginseng), Ginkgo (Ginkgo biloba), alho (Allium sativum) e Gengibre (Zingiber officinale), inibem a agregação plaquetária com potencial risco cirúrgico aos pacientes (DEXTRO MWB, et al., 2006).

Tanto os erros de conciliação que ocorrem na admissão hospitalar, quanto os que sucedem na alta, podem acarretar graves riscos à saúde do afetado. Entretanto, sob a óptica assistencial, os prejuízos advindos de 
falhas na conciliação de alta podem ser ainda maiores que os de vigência admissional, visto que durante a internação, o paciente permanece sob monitoramento ininterrupto da equipe multiprofissional, possibilitando que certos erros cometidos no ingresso do paciente, ainda sejam identificados e corrigidos no curso da internação (BORGES EP e MACEDO AF, 2011).

Contudo, se o paciente leva consigo, no retorno ao domicílio, erros de prescrição, torna-se integralmente vulnerável aos riscos de ineficácia, toxicidade e segurança farmacoterapêutica (CEBRIÁN BR, et al., 2016). Essa constatação evidencia a importância do farmacêutico realizar a conciliação de medicamentos na alta hospitalar e discutir, com a equipe médica, as discrepâncias identificadas, a fim de garantir que todos os medicamentos sejam prescritos em dose e padrão corretos.

Subsequentemente, pacientes e acompanhantes devem receber orientação farmacêutica, no que tange às interações fármaco-alimento, ao uso, armazenamento e acesso de medicamentos na rede pública de saúde e demais informações relevantes, por meio da elaboração de calendários posológicos, emprego de pictogramas, cartilhas informativas e outros recursos que colaborem para boa compreensão dos orientados, a fim de evitar recorrência de internações hospitalares decorrentes do uso inadequado de medicamentos. As instruções do farmacêutico devem ser fornecidas por escrito, preferencialmente impressas, visto que orientações verbais rumam facilmente ao esquecimento (MESQUITA E, et al., 2015).

É importante que outros membros da equipe multiprofissional sejam envolvidos na orientação ao paciente de alta. Os jejuns prolongados para fins de exames e procedimentos, a restrição alimentar, a inatividade física comuns das internações hospitalares, podem causar mudanças metabólicas e no sistema imunológico, que reduzem a força muscular e aumentam o risco de quedas, perigo que se acentua frente ao uso de determinados medicamentos, como opióides, antipsicóticos e benzodiazepínicos, que causam sedação e tontura, ou anti-hipertensivos e antiarrítmicos que induzem ortostatismo e redução da perfusão cerebral (REZENDE CP, et al., 2012).

A equipe de fisioterapia pode orientar pacientes e acompanhantes quanto aos cuidados que devem ser observados, no ambiente domiciliar, para prevenir lesões decorrentes de quedas. A orientação do nutricionista também é de suma importância, particularmente, para enfermos diabéticos, dislipêmicos e que utilizam medicamentos que sofrem interferência alimentar, como, por exemplo, a varfarina, anticoagulante antagonista de vitamina $\mathrm{K}$ (SOSA M e TABARÉ G, 2014).

\section{A contribuição do farmacêutico na identificação de problemas relativos à farmacoterapia}

A revisão das prescrições médicas constitui uma das atividades essenciais no cotidiano dos farmacêuticos hospitalares e contribui para que 0 uso dos medicamentos seja adequado e racional, com consequente aumento da segurança terapêutica do paciente (CRUZ TF, et al., 2019).

Uma pesquisa realizada por farmacêuticos clínicos em unidades de terapia intensiva e cardiologia de um hospital universitário terciário do Brasil constatou que $14,6 \%$ das prescrições revisadas apresentaram algum problema decorrente de interações medicamentosas, diluições, tempo de infusão, incompatibilidades, doses, intervalos entre doses ou vias de administração (REIS WCT, et al., 2013).

A revisão da prescrição propicia ao farmacêutico sugerir ajustes e discutir com médicos e enfermeiros alternativas para solucionar ou amenizar os problemas identificados e otimizar a terapia farmacológica (MIRANDA TMM, et al., 2012). Estudos mostram que grande parte dos erros relativos à farmacoterapia acontecem entre as etapas de prescrição e administração da medicação, o que oportuniza a contribuição dos farmacêuticos, já que os medicamentos só ficam, normalmente, disponíveis para uso, após serem dispensados pelo serviço de farmácia (REIS WCT, et al., 2013).

É importante que os farmacêuticos das unidades hospitalares elaborem, com base na literatura científica e auxílio dos outros membros da equipe multiprofissional de saúde, materiais de consulta, como manuais e protocolos, com informações de preparo, diluição, doses máximas e outros dados relevantes que agilizem o serviço farmacêutico de revisão das prescrições e sirvam de orientação para prescritores médicos (STORPIRTIS S, et al., 2003). 
Uma revisão sistemática de estudos que avaliaram a relevância das contribuições clínicas de farmacêuticos mostrou que, de modo geral, o envolvimento destes profissionais nas atividades clínicas melhorou os resultados referentes à prevenção de efeitos adversos e otimização farmacoterapêutica, com redução da mortalidade e dos custos com medicamentos (KABOLI PJ, 2006).

Um estudo que avaliou o impacto de intervenções farmacêuticas na redução de problemas relacionados à terapia medicamentosa, em um serviço especializado em cardiologia e doenças infecciosas, atestou que 0 envolvimento dos farmacêuticos reduziu significativamente os erros referentes ao uso de medicamentos. Além disso, os resultados mostraram que a maioria das intervenções estavam associadas a cardiologia e voltadas, principalmente, à farmacoterapia profilática do tromboembolismo venoso e ao uso de estatinas, betabloqueadores e inibidores da enzima conversora da angiotensina (IECA) (CHAMOUN NR, et al., 2016).

\section{O cuidado farmacêutico ambulatorial: prevenção e tratamento das DCVs}

A maioria das DCVs decorrem de fatores de risco modificáveis, que incluem o hábito do tabagismo, hipertensão e diabetes não controlados, sedentarismo, dieta alimentar hiperlipídica e obesidade, que embora sejam muito conhecidos, ainda não recebem a merecida importância por grande parte da população e dos portadores de DCVs, sendo assim, monitorados de forma deficitária (TSUYUKI RT, et al., 2016).

Quando devidamente treinado e capacitado, o farmacêutico comunitário pode colaborar na identificação e gestão dos fatores de risco cardiovascular, contribuindo para o aumento da qualidade de vida dos pacientes e redução no quantitativo de cirurgias, internações hospitalares e condições de inatividade física (SILVA EV, et al., 2008). Para que a terapia medicamentosa seja eficaz nos tratamentos de hipertensão, dislipidemias e diabetes e previna complicações cardiovasculares, além do uso adequado dos medicamentos, é importante que sejam praticadas condutas não farmacológicas, que implicam em mudanças de hábito. A educação faz parte dos cuidados em saúde, logo, o farmacêutico não deve abrir mão de ações educativas que visem reduzir os fatores de risco cardiovascular e melhorar os resultados da farmacoterapia (SILVA EV, et al., 2008).

Uma das mudanças de hábito que exibe relevância em termos de saúde cardiovascular é a cessação do tabagismo. Dados da Organização Mundial de Saúde (OMS) indicam que a prática tabágica ocasiona mais de sete milhões de mortes anualmente em todo o mundo e mostra relação com inúmeras doenças, dentre as quais se destacam as DCVs, os cânceres e as doenças respiratórias obstrutivas crônicas (GOYATÁ T, et al., 2014). As pesquisas salientam que o percentual de tabagistas que obtêm êxito ao tentar parar de fumar é maior quando há aconselhamento de profissionais de saúde (BRASIL, 2001; AMB/ANS, 2011).

O farmacêutico comunitário pode auxiliar na identificação das circunstâncias cotidianas que caracterizam "gatilhos" para a prática tabágica e no estabelecimento de estratégias que visem superar tais situações, com objetivo de cessar o tabagismo e prevenir recaídas. A partir de uma abordagem estruturada e de instrumentos específicos (REICHERT J, et al., 2008), pode-se avaliar o grau de dependência do paciente à nicotina, verificar se há indicação ou contraindicações para o tratamento farmacológico, classificar o grau motivacional do indivíduo na perspectiva de cessar o tabagismo e elaborar um plano de acompanhamento terapêutico para o paciente, podendo, inclusive, envolver outros profissionais de saúde, quando for necessário (BRASIL, 2013b). Antitabágicos a base de nicotina são medicamentos isentos de prescrição médica e podem ser prescritos, no Brasil, pelo farmacêutico (BRASIL, 2013a).

Uma outra abordagem que demanda orientação profissional e admite contribuição do farmacêutico comunitário é a orientação focada na prática sexual, visto que o tratamento das DCVs pode prejudicar a performance sexual dos pacientes, acarretando, muitas vezes, em redução da libido, disfunção erétil e depressão que podem ser oriundas do uso de medicamentos ou provenientes do temor dos doentes de sofrerem infarto agudo do miocárdio (IAM), dispnéia, crises de angina ou óbito durante a atividade sexual (STEIN R e HOHMANN CB, 2006). Os betabloqueadores adrenérgicos e as tiazidas, muito utilizados nos tratamentos de DCVs e hipertensão, associados a outros fatores, como tabagismo, diabetes e dislipidemias, podem desencadear disfunção erétil (PERDIGÃO C, et al., 2008).

O farmacêutico pode contatar os médicos responsáveis para discutir a possibilidade de substituir a farmacoterapia vigente por fármacos de outras classes (PERDIGÃO C, et al., 2008), além de aconselhar os 
pacientes sobre condutas que ajudem a reduzir esforço físico na atividade sexual e prevenir episódios de angina e IAM, tais como evitar relações sexuais após períodos de fadiga pronunciados, depois das refeições ou de consumir álcool (STEIN R e HOHMANN CB, 2006). Cabe ressaltar que o abandono do etilismo compõe uma das medidas não farmacológicas que devem ser estimuladas em pacientes de risco cardiovascular.

Como a interlocução entre o profissional de saúde e o paciente ainda pode enfrentar certos "tabus" em relação à sexualidade, principalmente em idosos, é possível que alguns indivíduos optem por tratar a disfunção erétil mediante automedicação, ficando, dessa forma, suceptíveis a prejuízos decorrentes de possíveis interações medicamentosas (STEIN R e HOHMANN CB, 2006).

O uso de inibidores da fosfodiesterase- 5 (sildenafila, tadalafila e vardenafila) combinado à terapia antianginosa com nitratos orgânicos constitui um exemplo de associação terapêutica que não deve ser utilizada, pois expõe o paciente ao risco de reação hipotensiva potencialmente fatal (PERDIGÃO C, et al., 2008). Entretanto, a administração de nitratos precedentemente à prática sexual pode ser benéfica, não somente na prevenção da dor precordial, como para reduzir o temor dos pacientes de sofrerem uma crise anginosa no trascurso da prática sexual, especialmente, quando a disfunção exibir motivação de cunho emocional (STEIN R e HOHMANN CB, 2006). Uma outra questão que desperta cada vez mais atenção das equipes multiprofissionais de saúde são os cuidados com a qualidade do sono. Os transtornos do sono reúnem um conjunto de condições clínicas potencialmente capazes de comprometer o sistema cardiovascular, contudo, o subdiagnóstico desses distúrbios ainda é muito frequente. A insônia se destaca como um dos transtornos do sono mais comuns na prática clínica (DRAGER LF, et al., 2018).

Estratégias que visem melhorar a qualidade do sono do doente, particularmente nos portadores de DCVs, colaboram para a qualidade de vida e melhoria do estado de saúde. Além de indicar medidas comportamentais, como higiene e restrição do sono, controle de estímulo e relaxamento, o farmacêutico pode ajudar o paciente, por meio da anamnese, a encontrar possíveis causas clínicas da insônia, que podem estar relacionadas à depressão, ansiedade, pânico noturno, tabagismo ou ingestão de bebidas cafeinadas, e encaminhá-lo ao tratamento adequado (RIBEIRO NF, 2016).

O manejo farmacológico da insônia é normalmente indicado quando as ações comportamentais são insuficientes ou o controle das condições clínicas associadas é insatisfatório (UFRGS, 2018). Vários agentes farmacológicos, como o hormônio melatonina, os moduladores dos receptores GABA-A (zolpidem, zaleplona, zopiclona), benzodiazepínicos e antidepressivos podem ser empregados na farmacoterapia da insônia, com potencialidade para efeitos adversos e interações medicamentosas que devem ser monitorados pelo médico e farmacêutico (RIBEIRO NF, 2016).

O emprego de fitoterápicos com propriedades sedativas, como Valeriana officinalis, Passiflora incarnata e Piper methysticum, aliado a medidas comportamentais, pode ser útil no tratamento da insônia de grau leve (BRASIL, 2016a). Alguns fitoterápicos são isentos de prescrição médica e podem ser prescritos, no Brasil, pelo farmacêutico (BRASIL, 2013a).

Tanto nas DCVs, como em outras doenças crônicas, a adesão ao tratamento é de suma importância para evitar descompensações ou insucesso da farmacoterapia. Na insuficiência cardíaca (IC), por exemplo, a falta de adesão constitui um dos principais problemas relacionados ao tratamento medicamentoso (LAFORESTE SKM, et al., 2013).

Assim, é importante que as orientações em saúde não abriguem lacunas ou dúvidas e que o acompanhamento farmacêutico seja individualizado, visando a implementação de medidas que evitem ou amenizem efeitos adversos e intolerâncias, além do estímulo às mudanças de hábito relevantes ao sucesso terapêutico. Quando necessário, o farmacêutico deve contatar o médico responsável para sugerir ajustes de doses ou substituição de medicamentos. Ensaios clínicos randomizados que avaliaram o impacto sobre os resultados a longo prazo dos cuidados farmacêuticos na IC mostraram reduções significativas nas taxas de todas as causas de hospitalização (ROBLEK T, et al., 2016).

Além disso, é importante que os portadores de DCVs sejam informados acerca de potenciais complicações que possam vir a surgir durante a evolução da doença, sobre os fatores de risco cardiovascular e a respeito 
da função e importância de cada medicamento que utilizam. Se o indivíduo não compreende bem o tratamento, pode não dar merecida importância às orientações que recebe.

Quanto às interações medicamentosas, o farmacêutico deve estar atento, visto que algumas associações colocam em risco a eficácia terapêutica e a segurança do paciente. Como muitos portadores de DCVs são polipatológicos e polimedicados, ficam susceptíveis a potenciais interações decorrentes do uso concomitante de medicamentos prescritos por médicos de diferentes especialidades. O uso prolongado de anti-inflamatórios não esteroidais, por exemplo, pode prejudicar o tratamento da hipertensão e de cardiopatias, em decorrência da possibilidade de provocar a redução do efeito de medicamentos diuréticos, betabloqueadores e IECA, favorecer a elevação da pressão arterial média e prejudicar a função renal (BATLOUNI M, 2010).

Com base na escolaridade, idade, polimedicação e outras características dos pacientes, o farmacêutico deve traçar estratégias que os ajudem a lembrar de administrar os medicamentos nos horários preestabelecidos, prevenir substituições acidentais e evitar doses duplicadas. Para isso, vale elaborar calendários posológicos, usar pictogramas ou cartilhas explicativas, registrar informes relevantes nas embalagens dos medicamentos e configurar dispositivos móveis com alarme para sinalizar o horário de administração dos medicamentos. É importante estimular o paciente a desenvolver autonomia e responsabilidade no autocuidado com a saúde, entretanto, quando isso não for possível, em decorrência do estado de saúde do assistido, familiares, vizinhos ou amigos, que possam auxiliá-lo, devem ser acionados.

\section{CONSIDERAÇÕES FINAIS}

Embora o aproveitamento clínico do farmacêutico nos serviços brasileiros de cardiologia ainda seja discreto, os estudos aqui apresentados evidenciam que a atuação deste profissional, tanto nas transições assistenciais, quanto nos cuidados oferecidos a pacientes internados e ambulatoriais, contribui para reduzir a incidência de erros referentes à prescrição e uso de medicamentos, otimizar a eficácia e segurança farmacoterapêuticas e melhorar a qualidade de vida dos pacientes. Contudo, é necessário investir na formação profissional do farmacêutico para que ofereça um serviço clínico eficiente e seguro aos assistidos.

\section{REFERÊNCIAS}

1. AMB/ANS - ASSOCIAÇÃO MÉDICA BRASILEIRA/AGÊNCIA NACIONAL DE SAÚDE SUPLEMENTAR. Diretrizes clínicas na saúde suplementar. Tabagismo, 2011, São Paulo.

2. BATLOUNI M. Anti-inflamatórios não esteroidais: efeitos cardiovasculares, cérebro-vasculares e renais. Arquivos Brasileiros de Cardiologia, 2010; 94(4):556-563.

3. BORGES EP, MACEDO AF. Omissão de fármacos modificadores do risco cardiovascular em idosos admitidos numa unidade de acidentes vasculares cerebrais. Revista Portuguesa de Clínica Geral, 2011; 27(2): 140-150.

4. BRANT LCC, et al. Variações e diferenciais da mortalidade por doença cardiovascular no Brasil e em seus estados, em 1990 e 2015: estimativas do estudo carga global de doença. Revista Brasileira de Epidemiologia, 2017; 20(1): 116-128.

5. BRASIL. Agência Brasileira de Vigilância Sanitária. Memento Fitoterápico. Farmacopéia Brasileira, Brasília, 2016a; 115p.

6. BRASIL. Conselho Federal de Farmácia. Serviços farmacêuticos diretamente destinados ao paciente, à família e à comunidade: contextualização e arcabouço conceitual. Brasília, 2016b; 200p.

7. BRASIL. Conselho Federal de Farmácia. Resolução n 586, de 21 de Julho de 2013. 2013a.

8. BRASIL. Ministério da Saúde. Abordagem e tratamento do fumante - Consenso. Instituto do Câncer. Rio de Janeiro, 2001.

9. BRASIL. Ministério da Saúde. Instituto Nacional do Câncer. Coordenação de ações estratégicas, Divisão de controle do tabagismo, 2013 - Protocolo Clínico e Diretrizes Terapêuticas de Dependência à Nicotina, Rio de Janeiro. 2013b.

10. CEBRIÁN BR, et al. Conciliación de la medicación en pacientes mayores de 75 anos. Revista de la Calidad Asistencial, 2016; 31: 29-35.

11. CHAMOUN NR; et al. Impact of clinical pharmacy interventions on medication error nodes. International Journal of Clinical Pharmacy, 2016; 38: 1436-1444.

12. CRUZ TF, et al. Análise do serviço de farmácia clínica em um hospital universitário. HURevista, 2019; 45(4): 408414. 
13. DEXTRO MWB, et al. Estudo da utilização no pré-operatório de medicamentos ou drogas fitoterápicas que alteram a coagulação sanguínea, 2006; 33(2): 107-110.

14. DRAGER LF, et al. $1^{\circ}$ Posicionamento brasileiro sobre o impacto dos distúrbios de sono nas doenças cardiovasculares da Sociedade Brasileira de Cardiologia. Arquivos Brasileiros de Cardiologia, 2018; 111(2): $290-340$.

15. DUNN SP, et al. The role of the clinical pharmacist in the care of patients with cardiovascular disease. Journal of the American College of Cardiology, 2015; 66(19): 2129-2139.

16. GARBERO RF, VIEIRA LA. Fármacos no pré-operatório. Revista do Hospital Universitário Pedro Ernesto. UERJ, 2007; 6: 29-37.

17. GOYATÁ T, et al. Impacto do programa de apoio ao tabagista de um município do sul de Minas Gerais, Brasil. Ciencia y Enfermeria, 2014; 20(1): 75-88.

18. JUVANY R, JÓDAR R. Importancia de la conciliacion de la medicacíon para garantizar la continuidad y la seguridad del proceso asistencial. Medicina Clinica, 2012; 139(15): 672-673.

19. KABOLI PJ, et al. Clinical pharmacists and inpatient medical care: a systematic review. Archives of Internal Medicine, 2006; 166: 955-964.

20. KUO GR, et al. Drug errors and related interventions reported by United States clinical pharmacists: The American College of Clinical Pharmacy Practice-Based Research Network Medication Error Detection, Amelioration and Prevention Study. Pharmacotherapy, 2013: 33 (3): 253-265.

21. LAFOREST SKM, et al. Clinical pharmacy services in heart failure: An opinion paper from the Heart Failure Society of America and American College of Clinical Pharmacy Cardiology Practice and Research Network. Journal of Cardiac Failure, 2013; 19(5): 354-369.

22. MACHUCA M, et al. 2004. Método dáder: manual de acompanhamento farmacoterapêutico.

23. MESQUITA E, et al. Síndrome pós-hospitalização: um novo desafio na prática cardiovascular. Arquivos Brasileiros de Cardiologia, 2015; 105(5): 540-544.

24. MIRANDA TMM, et al. Intervenções realizadas pelo farmacêutico clínico na unidade de primeiro atendimento. Einstein, 2012; 10(1): 74-78.

25. OPAS - ORGANIZAÇÃO PAN-AMERICANA DE SAÚDE. Revisado em 2017. Doenças cardiovasculares.

26. PERDIGÃO $C$, et al. Consensos sobre disfunção erétil - a disfunção erétil e a doença cardiovascular. Revista Portuguesa de Cardiologia, 2008; 27(1): 115-126

27. PEREIRA JC, et al. O perfil de saúde cardiovascular dos idosos brasileiros precisa melhorar: estudo de base populacional. Arquivos Brasileiros de Cardiologia, 2008; 91(1): 1-20.

28. REICHERT J, et al. Diretrizes para cessação do tabagismo. Jornal Brasileiro de Pneumologia, 2008; 34(10): 845-880.

29. REIS WCT, et al. Análise das intervenções de farmacêuticos clínicos em um hospital de ensino terciário do Brasil. Einsten, 2013; 11(2): 190-196.

30. REZENDE CP, et al. Queda entre idosos no Brasil e sua relação com o uso de medicamentos: revisão sistemática. Caderno de Saúde Pública, 2012; 28(12): 2223-2235.

31. RIBEIRO NF. Tratamento da insônia em atenção primária à saúde. Revista Brasileira de Medicina de Família e Comunidade, 2016; 11(38): 1-14.

32. ROBLEK T, et al. Clinical-pharmacist intervention reduces clinically relevant drug-drug interations in patients with heart failure: a radomized, double-blind, controlled trial. International Journal of Cardiology, 2016; 203: 647-652.

33. RUIZ BSJ, et al. Conciliación de la medicación al ingreso: resultados e identificación de pacientes diana. Revista de la Calidad Asistencial, 2016; 31: 36-44.

34. SÁNCHEZ OD. et al. Errones de conciliación en el ingreso y en el alta hospitalaria em pacientes ancianos polimedicados. Estudio prospectivo aleatorizado multicéntrico. Medicina Clínica, 2009; 133(19): 741-744.

35. SILVA EV, et al. O papel do farmacêutico comunitário no aconselhamento ao paciente. Farmacoterapêutica, 2008; 13(4-5): 1-3.

36. SOSA M, TABARÉ G. Warfarina: co-medicación y posibles interacciones. Farmacología Clínica, 2014; 9(3): 6-35.

37. STEIN R, HOHMANN CB. Atividade sexual e coração. Arquivos Brasileiros de Cardiologia, 2006; 86(1): 61-67.

38. STORPIRTIS S, et al. Novas diretrizes para assistência farmacêutica hospitalar: Atenção farmacêutica/Farmácia Clínica. In: GOMES MJVM, REIS AMM. Ciências Farmacêuticas: uma abordagem em Farmácia Hospitalar. São Paulo: Atheneu, 2003: 521-533.

39. UFRGS - UNIVERSIDADE FEDERAL DO RIO GRANDE DO SUL. Telecondutas: avaliação e manejo da insônia. Núcleo de Telessaúde da Universidade Federal do Rio Grande do Sul. Porto Alegre, 2018.

40. TSUYUKI RT, et al. The effectiveness of pharmacist interventions on cardiovascular risk. Journal of the American College of Cardiology, 2016; 67(24): 2846-2854. 